\title{
A prognostic model for stratification of stage IB/IIA esophageal squamous cell carcinoma: a retrospective study
}

Lei-Lei Wu ${ }^{1 \dagger}$, Qi-Long Ma ${ }^{2+}$, Wei Huang ${ }^{1 \dagger}$, Xuan Liu' ${ }^{1}$, Li-Hong Qiư ${ }^{1}$ Peng Lin ${ }^{1}$, Hao Long ${ }^{1}$, Lan-Jun Zhang ${ }^{1}$ and Guo-Wei Ma ${ }^{1,3^{*}}$ (D)

\begin{abstract}
Background: To explore the postoperative prognosis of esophageal squamous cell carcinoma (ESCC) patients with stage IB/IIA, using a prognostic score (PS).

Methods: Stage IB/IIA ESCC patients who underwent esophagectomy from 1999 to 2010 were included. We retrospectively recruited 153 patients and extracted their medical records. Moreover, we analyzed the programmed death ligand-1 (PD-L1) expression of their paraffin tissue. The cohort were randomly divided into a training group $(\mathrm{N}=123)$ and a validation group $(\mathrm{N}=30)$. We selected overall survival $(\mathrm{OS})$ as observed endpoint. Prognostic factors with a multivariable two-sided $P<0.05$ met standard of covariate inclusion.

Results: Univariable and multivariable analyses identified pTNM stage, the number of lymph nodes (NLNs) and PD-L1 expression as independent OS predictors. Primary prognostic score which comprised above three covariates adversely related with OS in two cohorts. PS discrimination of OS was comparable between the training and internal validation cohorts (C-index $=0.774$ and 0.801 , respectively). In addition, the PS system had an advantage over pTNM stage in the identification of high-risk patients (C-index $=0.774$ vs. C-index $=0.570, P<0.001)$. Based on PS cutoff, training and validation datasets generated low-risk and high-risk groups with different OS. Our three-factor PS predicted OS (low-risk subgroup vs. high-risk subgroup 60-month OS, 74\% vs. 23\% for training cohort and 83\% vs. 45\% for validation cohort).
\end{abstract}

Conclusion: Our study suggested a PS for significant clinical stratification of IB/IIA ESCC to screen out subgroups with poor prognosis.

Keywords: Esophageal squamous cell carcinoma, Stage IB/IIA, Lymph nodes, PD-L1, Prognosis

\section{Background}

Esophageal carcinoma (EC) is a worldwide malignancy, ranking 9th and 6th in terms of incidence and mortality, respectively. About 509,000 EC cases die every year, and

\footnotetext{
*Correspondence: magw@sysucc.org.cn

'Lei-Lei Wu, Qi-Long Ma and Wei Huang contributed equally to this work

${ }^{3}$ The Department of Thoracic Surgery, Sun Yat-Sen University Cancer

Center, 651 Dongfengdong Road, Guangzhou 510060, People's Republic of China

Full list of author information is available at the end of the article
}

its major histological subtype is esophageal squamous cell carcinoma (ESCC) [1-4]. Over fifty per cent of new EC cases occur in China [5, 6], causing this country to present the highest mortality rate, with ESCC accounting for over 90\% [6]. EC treatments include surgery, radiotherapy, chemotherapy, and immunotherapy, among which surgery remains the main treatment. Unfortunately, in China, the prognosis of surgical EC resection remains poor, with a 5 -year survival rate of only $20-40 \%$ 
after esophagectomy [7]. Therefore, it's important to screen out the patients with poor prognosis.

Both TNM stage and the number of lymph nodes (NLNs) dissected in surgery have been presented with clinical prognosis indicator in esophageal cancer [8]. In addition, there are still few predictors of EC development and prognosis [9-14]. Several previous studies reported that the expression of programmed death ligand-1(PDL1) in lung cancer, breast cancer, and other tumors has a relation with the clinical significance of patients [1522]. PD-L1 is a member of the B7-CD28 family, which is related to the tumor cell immune escape, playing an important role in induced $\mathrm{T}$ cell apoptosis $[15,21]$.

Here, we constructed a prognostic score (PS) system based on the TNM stage, NLNs, and expression of PD-L1, and they were independent prognostic indictors for OS. The current PS was able to divide the cohort into low- and high-risk subgroups, according to the survival outcome. This might provide clinically applicable information to give recommendations of follow-up management and monitoring.

\section{Methods \\ Patients}

The Ethics Committee of Sun Yat-sen University Cancer Center (SYSUCC) approved the study's protocol and exempted informed consent (approval number: YB2016070). A total of 153 patients who underwent esophagectomy at the Department of Thoracic Surgery of SYSUCC between May 1999 and October 2010 were retrospectively enrolled in our study. Eligible cases had stage IB/ IIA ESCC, pathologically confirmed according to the $8^{\text {th }}$ edition of the American Joint Committee on Cancer
(AJCC) Staging Manual. The following exclusion criteria were applied: (1) patients who had received adjuvant and neoadjuvant cytotoxic chemotherapy or radiotherapy or immunotherapy regimens; (2) patients with a history of another malignant tumor; (3) patients with incomplete resection or margin residual tumor cells; (4) patients who died from postoperative complications or died within 1 month; (5) patients whose primary tumors were in the cervical esophagus or esophagogastric junction; and (6) patients with other pathological subtypes of EC besides ESCC. Included patients did not obviously present clinical evidence of inflammatory conditions. The pathological staging was translated into the $8^{\text {th }}$ edition of AJCC, using the patients' records. The diagram of the study was presented with Fig. 1.

\section{Surgery}

Surgeries were performed according to the following standard approaches of esophagectomy: McKeown (laparotomy, right thoracotomy, and neck incision), the Sweet (diaphragm incision and left thoracotomy), and the Ivor Lewis (right thoracotomy and laparotomy) procedures. Within the cohort, patients all performed thoracoabdominal dissection of lymph nodes. During surgery, the mean number of dissected lymph nodes (LNs) was 19.7.

\section{Follow-up}

The median follow-up time was 97.9 months, with the last follow-up session being performed in May 2019. The patients were recommended to visit the outpatient department for follow-up every 3-6 months for the first 2 years, every 6-12 months for the next 3 years, and every year thereafter. The barium esophagography and


Fig. 1 The diagram of this study 
neck-abdomen CT scans constituted the major followup examinations. Patients might undergo positron emission tomography-CT and/or endoscopy, as necessary.

\section{Immunohistochemical staining}

Tumor and non-tumor paraffin tissues of all 153 patients were performed according to an Envision system of manufacturer's instructions (Glostrup, Dako, Denmark). Polyclonal rabbit PD-L1 antibody (1:100; Cell Signaling Technology, Beverly, MA) and Ventana OmniMap anti-rabbit antibody were used as the primary and secondary antibodies, respectively. Staining intensity and extent were scored $0-3$ and $0-4$, respectively $(0 \%$ for $0 ; 1-10 \%$ for 1 point; $11-25 \%$ for 2 points; $26-40 \%$ for three points; $>41 \%$ for 4 points). For each staining, the final quantitation was obtained by multiplying the two scores. The results of immunohistochemical staining were interpreted independently by two pathologists under double-blind conditions. They didn't know any clinical and other pathological information. If the results were inconsistent, they would perform a joint discussion to decide the final result.

\section{Statistical analysis}

Statistical analyses were performed using $\mathrm{R}$ version 3.5.2 (https://www.r-project.org/) and the SPSS Statistics 25.0 software (IBM SPSS, Inc., Chicago, IL, USA). The hazard ratio (HR) with $95 \%$ confidential interval $(\mathrm{CI})$ were calculated by multivariate regression analysis. The cutoff value of PD-L1 expression and NLNs were determined using median, 3.0 and 16.0, respectively. According to above cutoff values, the PD-L1 expression $\leq 3$ was regarded as low expression, and $>3$ was defined as high expression. The associations between the PD-L1 expression, NLNs, and clinicopathological factors were assessed using the student's $t$ test, $\chi^{2}$ test and Fisher exact test. Standard error (SE) and standard deviation (SD)were used to evaluate the stability of continuous variables. Univariable analysis was performed to evaluate the influence of differentiation, pathological $\mathrm{T}$ stage, sex, pathological TNM stage, age, NLNs, smoking history, tumor length, drinking history, surgical approach, lymph node dissection of left recurrent laryngeal nerve, lymph node dissection of right recurrent laryngeal nerve, dissection of left gastric artery lymph node, dissection of subcarinal lymph node, and the level of PD-L1 expression on OS. A two-sided $P<0.05$ was considered statistically significant. Multivariable analysis was used to select independent factors affecting OS. Variables were selected with univariable analysis of $P<0.05$. In this study, we used one-way ANOVA test, linear regression and Pearson's correlation analysis to explore the association between pathological TNM stage, NLNs and PD-L1 expression. The log-rank tests and Kaplan-Meier analysis were used to compare survival curves between groups. The model was developed and validated using a randomized method to extract trained and validated datasets. We used the function of "Random Sample of Cases" in SPSS, and set random sample size as 30 . This randomized method made the ratio of training group to validation group 4:1.

Patients' clinical characteristics and demographics were reported for the training group. The PS system for OS was constructed using three factors (NLNs, pTNM stage, and the expression of PD-L1), which was derived from the training dataset. The cohort was divided into a low-risk and a high-risk subgroup using median determine the PS cutoff value in the training cohort. A same cutoff value of risk score was defined to classify the patients in the internal validation cohort. C-index was used to estimate the discrimination of the multivariable survival prognostic model.

In the validation cohort, PS was applied to calculate the risk score, and classified patients into two subgroups, the low- and high-risk subgroups, basing on the same cutoff values defined in the training dataset.

\section{Results}

The clinical variables of patients in the training and internal validation cohorts were shown in Table 1 . Among the 153 patients, the 1-, 3- and 5-year OS rates were $84.0 \%$, $71.0 \%$ and $46.0 \%$, respectively. The patients' age ranged from 37 to 81 years old (median, 60 years old). In the training group, the 1-, 3- and 5-year OS rates were $82.0 \%$, $70.0 \%$ and $45.0 \%$, respectively, and the median and mean survival times from surgery to the last censoring date were 91.9 and 82.0 months, respectively.

Within the training cohort, a high level of PD-L1 expression was found in 58 of the 123 (47.2\%) cases, and the expression of PD-L1 was shown as Fig. 2. The significance of PD-L1 and NLNs in ESCC was verified by correlating the status of PD-L1 and NLNs in 123 ESCC cases with widely recognized clinicopathological features (Table 2). Our results suggest that NLNs is correlated with surgical approach (Table 2). Univariable and multivariable analyses were performed to identify correlations between clinical characteristics and OS. As shown in Table 3, univariable and multivariable analyses identified the following clinical factors as significant OS prognostic indictors in patients with ESCC: NLNs (adjusted HR $0.963,95 \%$ CI $0.938-0.989, P=0.006)$, pTNM stage (adjusted HR 1.987, 95\%CI 1.050-3.761, $P=0.035$ ), and the expression of PD-L1 (adjusted HR 4.746, 95\%CI 2.669-8.438, $P<0.001)$. The association of above three factors was shown in Fig. 3. We found that there was no statistically significant correlation among NLNs, pTNM stage, and the expression of PD-L1. In addition, our study 
Table 1 The clinicopathologic characteristics of patients in the training and validation cohorts

\begin{tabular}{|c|c|c|c|c|c|c|}
\hline \multirow[t]{2}{*}{ Variable } & \multirow{2}{*}{$\begin{array}{l}\text { All cohort } \\
\mathrm{N}=153(\%)\end{array}$} & \multicolumn{2}{|c|}{ Training cohort } & \multicolumn{2}{|c|}{ Validation cohort } & \multirow[t]{2}{*}{ Pvalue } \\
\hline & & $N=123$ & $\%$ & $\mathrm{~N}=30$ & $\%$ & \\
\hline Sex & & & & & & $0.248^{*}$ \\
\hline Male & $115(75.2)$ & 90 & 73.2 & 25 & 83.3 & \\
\hline Female & $38(24.8)$ & 33 & 26.8 & 5 & 16.7 & \\
\hline Age (year, mean $\pm S D$ ) & $59.7 \pm 8.9$ & $59.5 \pm 9.0$ & & $60.5 \pm 8.8$ & & $0.583^{* *}$ \\
\hline Smoking status & & & & & & $0.157^{*}$ \\
\hline No & $58(37.9)$ & 50 & 40.7 & 8 & 26.7 & \\
\hline Yes & $95(62.1)$ & 73 & 59.3 & 22 & 73.3 & \\
\hline Drinking status & & & & & & $0.379^{*}$ \\
\hline No & $107(69.9)$ & 88 & 71.5 & 19 & 63.3 & \\
\hline Yes & $46(30.1)$ & 35 & 28.5 & 11 & 36.7 & \\
\hline Tumor length $(\mathrm{cm}$, mean $\pm \mathrm{SD})$ & $3.6 \pm 1.5$ & $3.7 \pm 1.7$ & & $3.3 \pm 1.0$ & & $0.227^{* *}$ \\
\hline PD-L1 expression & & & & & & $0.780^{*}$ \\
\hline Low & $80(52.3)$ & 65 & 52.8 & 15 & 50.0 & \\
\hline High & $73(47.7)$ & 58 & 47.2 & 15 & 50.0 & \\
\hline Surgery approach & & & & & & $0.156^{*}$ \\
\hline Left thorax & $108(70.6)$ & 90 & 73.2 & 18 & 60.0 & \\
\hline Right thorax & $45(29.4)$ & 33 & 26.8 & 12 & 40.0 & \\
\hline Differentiation & & & & & & $0.013^{*}$ \\
\hline Well-moderate & $71(46.4)$ & 51 & 41.5 & 20 & 66.7 & \\
\hline Poor & $82(53.6)$ & 72 & 58.5 & 10 & 33.3 & \\
\hline pT stage & & & & & & $0.549^{*}$ \\
\hline $\mathrm{T} 2$ & $59(38.6)$ & 46 & 37.4 & 13 & 43.3 & \\
\hline $\mathrm{T} 3$ & $94(61.4)$ & 77 & 62.6 & 17 & 56.7 & \\
\hline NLNs (mean \pm SD) & $19.7 \pm 14.1$ & $19.3 \pm 13.9$ & & $21.4 \pm 14.9$ & & $0.470^{* *}$ \\
\hline pTNM stage & & & & & & $0.102^{*}$ \\
\hline $\mathrm{IB}$ & $52(34.0)$ & 38 & 30.9 & 14 & 46.7 & \\
\hline$\| \mathrm{A}$ & $101(66.0)$ & 85 & 69.1 & 16 & 53.3 & \\
\hline LNs dissection of left recurrent laryngeal nerve & & & & & & $0.592^{*}$ \\
\hline No & $47(30.7)$ & 39 & 31.7 & 8 & 26.7 & \\
\hline Yes & $106(69.3)$ & 84 & 68.3 & 22 & 73.3 & \\
\hline LNs dissection of right recurrent laryngeal nerve & & & & & & $0.415^{*}$ \\
\hline No & $45(29.4)$ & 38 & 30.9 & 7 & 23.3 & \\
\hline Yes & $108(70.6)$ & 85 & 69.1 & 23 & 76.7 & \\
\hline Dissection of left gastric artery LNs & & & & & & $1.00^{* * *}$ \\
\hline No & $9(5.9)$ & 7 & 5.7 & 2 & 6.7 & \\
\hline Yes & $144(94.1)$ & 116 & 94.3 & 28 & 93.3 & \\
\hline Dissection of subcarinal LNs & & & & & & $0.69^{* * *}$ \\
\hline No & $10(6.5)$ & 9 & 7.3 & 1 & 3.3 & \\
\hline Yes & $143(93.5)$ & 114 & 92.7 & 29 & 96.7 & \\
\hline
\end{tabular}

NLNs, the number of lymph nodes; LNs, lymph nodes

${ }^{*} P$ value was calculated by $X^{2}$ test; ${ }^{* *} P$ value was calculated by student's $t$ test; ${ }^{* * *}$ Fisher exact test

showed that the level of PD-L1 expression, NLNs, and pTNM stage were significantly associated with OS in patients of ESCC.

Based on the results of the training cohort information analysis, we constructed the PS system and tested the covariates listed in Table 4 for their relation with OS. The PS system was based on weighting (derived by the $\beta$-coefficient of the respective $\log [\mathrm{HRs}]$ ) of the three significant covariates in the training group (Table 4), which generated C-index of $0.774 \pm 0.029$ for OS. In fact, in the 
Patients included in this study (SYSUCC)

(1) Undergone esophagectomy

(2) Histopathologically diagnosed as ESCC

(3) Stage IB/IIA (8 $8^{\text {th }}$ AJCC)

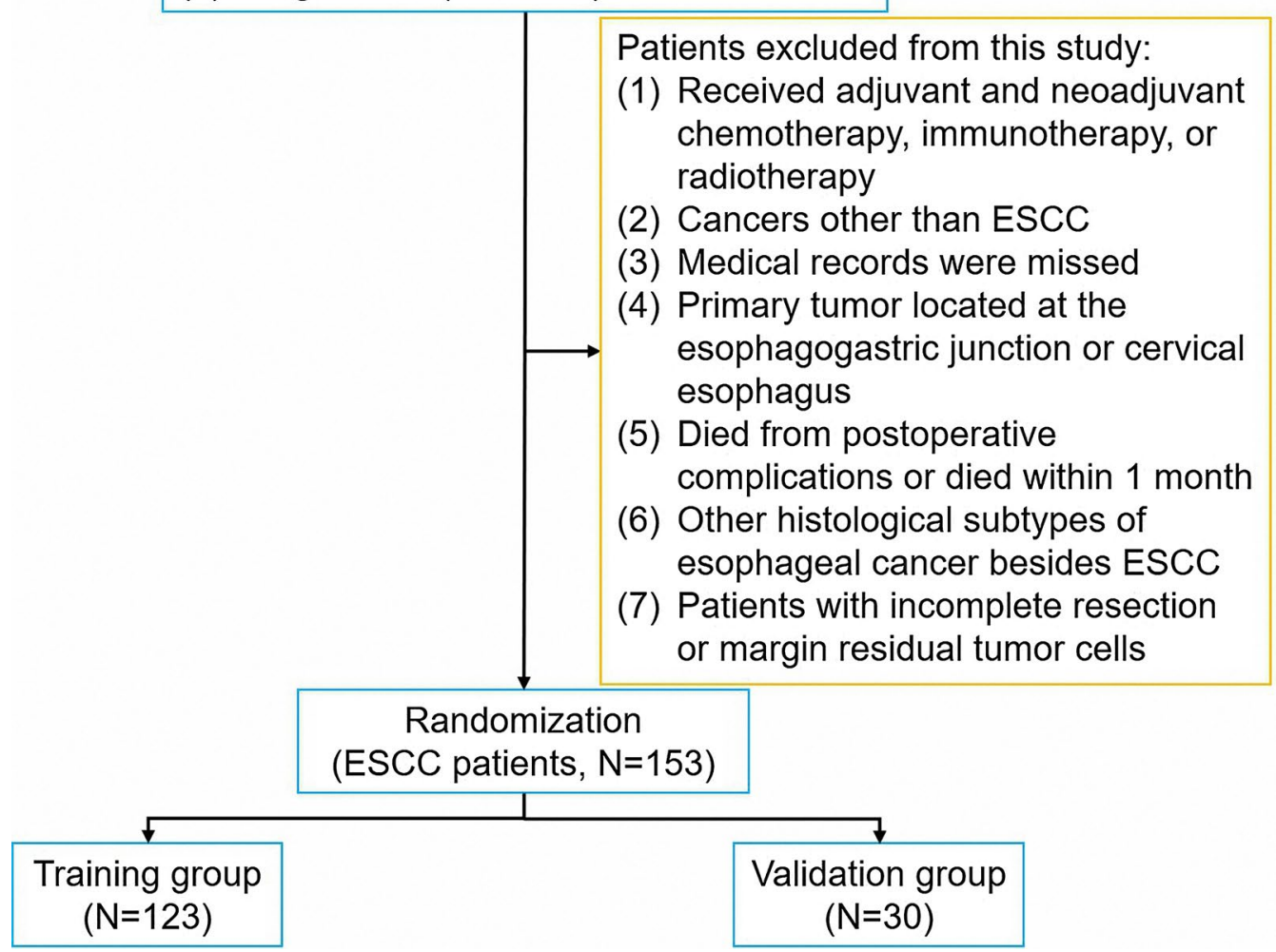

Fig. 2 Immunohistochemical staining of PD-L1 in ESCC paraffin tissue with stage IB/IIA (a Low expression of PD-L1, staining intensity score was 1 point, staining extent score was 1 point, final score was 1 point; $\mathbf{b}$ High expression of PD-L1, staining intensity score was 3 points, staining extent score was 2 points, final score was 6 points)

training group, our PS included pTNM stage, NLNs and the expression of PD-L1 had a more exact predictive ability than pTNM stage for 5 -year OS (PS: C-index $=0.774$, TNM stage: $\mathrm{C}$-index $=0.570, P<0.0001)$. In other words, the PS system had an advantage over pTNM stage in the discrimination of high-risk patients. This model allowed us to define a low-risk subgroup presenting a significantly increased likelihood of survival (unadjusted HR 6.195, 95\% CI, 3.368-11.396; $P^{<} 0.001$, Fig. 4a). The PS cutoff value was determined to distinguish between the highrisk and low-risk subgroups, using the median 107.0.

In the validation group, the 1-, 3- and 5-year OS rates were $93.0 \%, 77.0 \%$ and $54.0 \%$, respectively, and the median and mean survival times were 98.2 and 94.1 months, respectively. To validate the PS's predictive accuracy for OS in IB/IIA ESCC, we examined the PS in the internal validation cohort: a cohort of 30 cases. The same PS cutoff value of 107.0 allowed us to stratify the patients within the validation cohort into either a low-risk subgroup with a significantly better OS or a high-risk subgroup (unadjusted HR 6.766, 95\% CI, 1.45031.564; $P=0.005$, Fig. $4 \mathrm{~b}$ ). The PS in the internal validation dataset yielded C-index of $0.801 \pm 0.061$ for OS.

\section{Discussion}

IB/IIA stage ESCC is the disease without metastases of lymph nodes, which is seen as early stage disease. Guidelines doesn't recommend that these postoperative patients need to undergo the adjuvant treatment, such as chemotherapy and radiotherapy. However, the occurrence and development of ESCC is complex, and prognosis in a part of postoperative ESCC patients with stage IB/IIA is poor. The present study aimed to provide useful information to screen out the patients with poor prognosis. The patients' clinical information and immunohistochemistry were analyzed, including the indicators shown in Table 1. Three meaningful indicators, NLNs, pTNM stage and PD-L1 expression levels, were selected through 
Table 2 Correlation between PD-L1 expression, NLNs and clinicopathological characteristics in training cohort

\begin{tabular}{|c|c|c|c|c|c|c|}
\hline \multirow[t]{3}{*}{ Variable } & \multicolumn{2}{|c|}{ PD-L1 expression } & \multirow[t]{3}{*}{ Pvalue } & \multicolumn{2}{|l|}{ NLNs } & \multirow[t]{3}{*}{ Pvalue } \\
\hline & Low & High & & $\leq 16$ & $>16$ & \\
\hline & $\mathrm{N}=65(\%)$ & $\mathrm{N}=58(\%)$ & & $N=66(\%)$ & $\mathrm{N}=57(\%)$ & \\
\hline Sex & & & $0.819^{*}$ & & & $0.773^{*}$ \\
\hline Male & $47(72.3)$ & $43(74.1)$ & & $49(74.2)$ & $41(71.9)$ & \\
\hline Female & $18(27.7)$ & $15(25.9)$ & & $17(25.8)$ & $16(28.1)$ & \\
\hline Age (years, mean $\pm S D$ ) & $59.5 \pm 8.9$ & $59.5 \pm 9.1$ & $0.980^{* *}$ & $60.0 \pm 7.9$ & $59.0 \pm 10.0$ & $0.544^{* *}$ \\
\hline Smoking status & & & $0.832^{*}$ & & & $0.424^{*}$ \\
\hline No & $27(41.5)$ & $23(39.7)$ & & $29(43.9)$ & $21(36.8)$ & \\
\hline Yes & $38(58.5)$ & $35(60.3)$ & & $37(56.1)$ & $36(63.2)$ & \\
\hline Drinking status & & & $0.549^{*}$ & & & $0.625^{*}$ \\
\hline No & $48(73.8)$ & $40(69.0)$ & & $46(69.7)$ & $42(73.7)$ & \\
\hline Yes & $17(26.2)$ & $18(31.0)$ & & $20(30.3)$ & $15(26.3)$ & \\
\hline Tumor length $(\mathrm{cm}$, mean $\pm \mathrm{SD})$ & $3.8 \pm 1.6$ & $3.6 \pm 1.7$ & $0.440^{* *}$ & $3.6 \pm 1.5$ & $3.8 \pm 1.8$ & $0.584^{* *}$ \\
\hline Surgical approach & & & $0.525^{*}$ & & & $0.002^{*}$ \\
\hline Left thorax & $46(70.8)$ & 44 (75.9) & & $56(84.8)$ & $34(59.6)$ & \\
\hline Right thorax & $19(29.2)$ & $14(24.1)$ & & $10(15.2)$ & $23(40.4)$ & \\
\hline NLNs & & & $0.077^{*}$ & & & - \\
\hline$\leq 16$ & $30(46.2)$ & $36(62.1)$ & & - & - & \\
\hline$>16$ & $35(53.8)$ & 22 (37.9) & & - & - & \\
\hline Differentiation & & & $0.701^{*}$ & & & $0.385^{*}$ \\
\hline Well-moderate & $28(43.1)$ & $23(29.7)$ & & 25 (37.9) & $26(45.6)$ & \\
\hline Poor & $37(56.9)$ & $35(60.3)$ & & $41(62.1)$ & $31(54.4)$ & \\
\hline pT stage & & & $0.796^{*}$ & & & $0.215^{*}$ \\
\hline $\mathrm{T} 2$ & $25(38.5)$ & $21(36.2)$ & & $28(42.4)$ & 18 (31.6) & \\
\hline $\mathrm{T} 3$ & $40(61.5)$ & $37(63.8)$ & & $38(57.6)$ & $39(68.4)$ & \\
\hline pTNM stage & & & $0.254^{*}$ & & & $0.811^{*}$ \\
\hline $\mathrm{IB}$ & $23(35.4)$ & 15 (25.9) & & $21(31.8)$ & $17(29.8)$ & \\
\hline$\| \mathrm{A}$ & $42(64.6)$ & $43(74.1)$ & & $45(68.2)$ & $40(70.2)$ & \\
\hline PD-L1 expression & & & - & & & $0.077^{*}$ \\
\hline Low & - & - & & $30(45.5)$ & $35(61.4)$ & \\
\hline High & - & - & & $36(54.5)$ & $22(38.6)$ & \\
\hline LNs dissection of left recurrent laryngeal nerve & & & $0.813^{*}$ & & & $0.454^{*}$ \\
\hline No & $20(30.8)$ & $19(32.8)$ & & $19(28.8)$ & $20(35.1)$ & \\
\hline Yes & $45(69.2)$ & $39(67.2)$ & & $47(71.2)$ & $37(64.9)$ & \\
\hline LNs dissection of right recurrent laryngeal nerve & & & $0.416^{*}$ & & & $0.586^{*}$ \\
\hline No & $18(27.7)$ & $20(34.5)$ & & $19(28.8)$ & $19(33.3)$ & \\
\hline Yes & $47(72.3)$ & $38(65.5)$ & & $47(71.2)$ & $38(66.7)$ & \\
\hline Dissection of left gastric artery LNs & & & $0.706^{* * *}$ & & & $0.121^{* * *}$ \\
\hline No & $3(4.6)$ & $4(6.9)$ & & $6(9.1)$ & $1(1.8)$ & \\
\hline Yes & $62(95.4)$ & $54(93.1)$ & & $66(90.9)$ & $56(98.2)$ & \\
\hline Dissection of subcarinal LNs & & & $0.498^{* * *}$ & & & $0.732^{* * *}$ \\
\hline No & $6(9.2)$ & $3(5.2)$ & & $4(6.1)$ & $5(8.8)$ & \\
\hline Yes & $59(90.8)$ & $55(94.8)$ & & 62 (93.9) & $52(91.2)$ & \\
\hline
\end{tabular}

NLNs, the number of lymph nodes

${ }^{*} P$ value was calculated by $X^{2}$ test; ${ }^{* *} P$ value was calculated by student's $t$ test; ${ }^{* * *}$ Fisher exact test 
Table 3 Univariable and multivariable Cox proportional hazard regression analyses of the characteristics in training cohort

\begin{tabular}{|c|c|c|c|c|c|c|}
\hline \multirow[t]{2}{*}{ Variables } & \multicolumn{3}{|c|}{ Univariable analysis } & \multicolumn{3}{|c|}{ Multivariable analysis } \\
\hline & $H R$ & $95 \% \mathrm{Cl}$ & $P$ value & $H R$ & $95 \% \mathrm{Cl}$ & $P$ value \\
\hline \multicolumn{7}{|l|}{ Sex } \\
\hline Male & 1 (reference) & & & & & \\
\hline Female & 1.170 & $0.665-2.061$ & 0.586 & & & \\
\hline Age (year, continuous) & 1.024 & $0.995-1.053$ & 0.107 & & & \\
\hline \multicolumn{7}{|l|}{ Smoking status } \\
\hline No & 1 (reference) & & & & & \\
\hline Yes & 1.305 & $0.764-2.231$ & 0.330 & & & \\
\hline \multicolumn{7}{|l|}{ Drinking status } \\
\hline No & 1 (reference) & & & & & \\
\hline Yes & 1.627 & $0.949-2.790$ & 0.077 & & & \\
\hline \multicolumn{7}{|l|}{ Differentiation } \\
\hline Well-moderate & 1 (reference) & & & & & \\
\hline Poor & 0.681 & $0.406-1.140$ & 0.144 & & & \\
\hline \multicolumn{7}{|l|}{ pT stage } \\
\hline $\mathrm{T} 2$ & 1 (reference) & & & & & \\
\hline $\mathrm{T} 3$ & 1.677 & $0.952-2.954$ & 0.073 & & & \\
\hline \multicolumn{7}{|l|}{ pTNM stage } \\
\hline $\mathrm{IB}$ & 1 (reference) & & & 1 (reference) & & \\
\hline$\| \mathrm{A}$ & 2.080 & $1.101-3.930$ & 0.024 & 1.987 & $1.050-3.761$ & 0.035 \\
\hline Tumor length (cm, continuous) & 1.031 & $0.889-1.197$ & 0.684 & & & \\
\hline \multicolumn{7}{|l|}{ Surgery approach } \\
\hline Left & 1 (reference) & & & & & \\
\hline Right & 0.624 & $0.330-1.179$ & 0.146 & & & \\
\hline NLNs (continuous) & 0.960 & $0.935-0.985$ & 0.002 & 0.963 & $0.938-0.989$ & 0.006 \\
\hline \multicolumn{7}{|l|}{ PD-L1 expression } \\
\hline Low & 1 (reference) & & & 1 (reference) & & \\
\hline High & 4.910 & $2.770-8.702$ & $<0.001$ & 4.746 & $2.669-8.438$ & $<0.001$ \\
\hline \multicolumn{7}{|l|}{ Dissection of left gastric artery LNs } \\
\hline No & 1 (reference) & & & & & \\
\hline Yes & 4.338 & $0.599-31.44$ & 0.146 & & & \\
\hline \multicolumn{7}{|l|}{ Dissection of subcarinal LNs } \\
\hline No & 1 (reference) & & & & & \\
\hline Yes & 0.965 & $0.349-2.668$ & 0.946 & & & \\
\hline \multicolumn{7}{|c|}{ LNs dissection of left recurrent laryngeal nerve } \\
\hline No & 1 (reference) & & & & & \\
\hline Yes & 1.362 & $0.756-2.452$ & 0.304 & & & \\
\hline \multicolumn{7}{|c|}{ LNs dissection of right recurrent laryngeal nerve } \\
\hline No & 1 (reference) & & & & & \\
\hline Yes & 1.128 & $0.634-2.008$ & 0.681 & & & \\
\hline
\end{tabular}

Multivariable analysis's method is Forward: LR

$\mathrm{HR}$, hazard ratio; $\mathrm{Cl}$, confidential interval; NLNs, the number of lymph nodes; LNs, lymph nodes

univariable and multivariable analyses of the training set. We constructed a prognostic model based on NLNs, pTNM stage and PD-L1 expression and successfully identified high- and low-risk populations within the training and validation cohorts. Our model has a significant effect on patients' differentiation (Fig. 4), as the C-index predicting the OS rate reaches 0.801 and 0.774 in the internal validation and training sets, respectively. In fact, in the training group, our PS had a significant improvement than pTNM stage for predictive ability of 5-year OS (PS: 

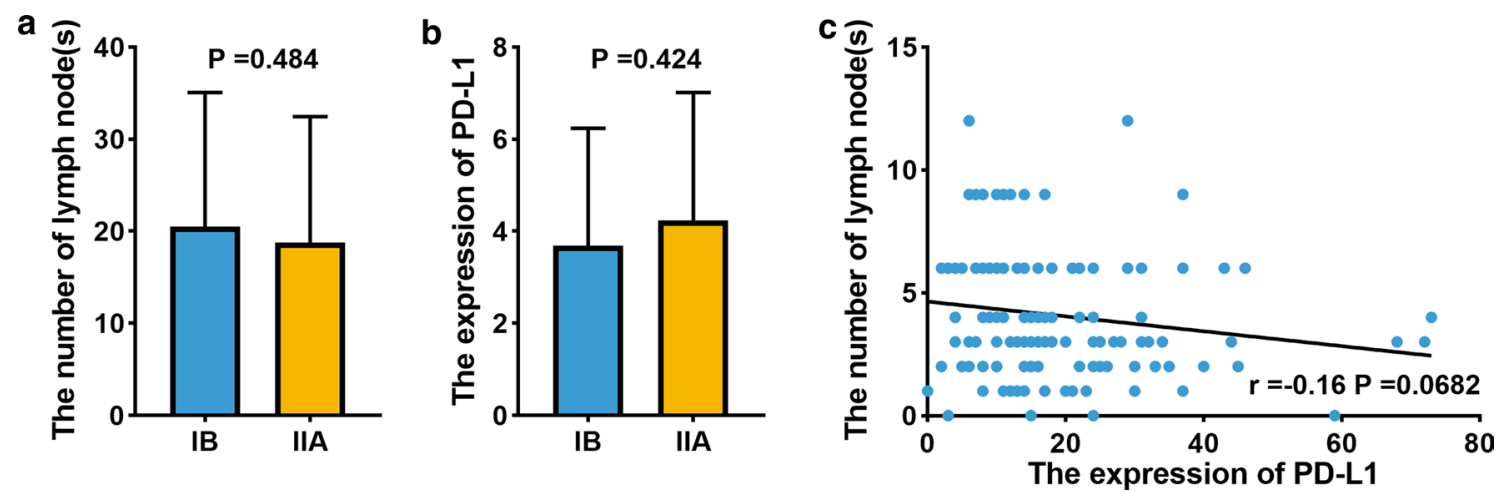

Fig. 3 a Correlation between TNM stage and the number of lymph nodes (NLNs) (one-way ANOVA test); $\mathbf{b}$ Correlation between TNM stage and PD-L1 expression (one-way ANOVA test); C. Correlation between NLNs and PD-L1 expression (linear regression and Pearson's correlation analysis)

Table 4 Constructed prognostic score to predict overall survival in stage IB/IIA ESCC patients

\begin{tabular}{lll}
\hline Covarite & $\boldsymbol{\beta}[\mathbf{H R}=\exp (\boldsymbol{\beta})]$ & Score \\
\hline NLNs & -0.038 & $-0.038^{*}$ continuous \\
PDL-1 expression & 1.557 & $\begin{array}{c}1.557^{*}(\text { low expres- } \\
\text { sion }=0, \text { high expres- } \\
\text { sion }=1)\end{array}$ \\
& & $0.687^{*}(\mathrm{IB}=1, \| \mathrm{A}=2)$ \\
pTNM stage & 0.687 & total score ${ }^{*} 100$ \\
Total computed score & & \\
and risk stratification & & $\leq 107.0$ \\
Low risk & & $>107.0$ \\
High risk & &
\end{tabular}

NLNs, the number of lymph nodes
C-index $=0.774$, TNM stage: $\mathrm{C}$-index $=0.570, P<0.0001)$. Of note, the PS system had an advantage over pTNM stage in the discrimination of high-risk patients. According to our findings, patients with high risk score might require close attention from doctors and they would better be recommended to choose a shorter follow-up interval from what guidelines suggest [23]. In terms of clinical application, routine postoperative pathological records include tumor invasion, and NLNs. Evaluation of PD-L1 expression requires only immunohistochemical staining of postoperative paraffin tissues and the respective interpretation, independently performed by two pathologists. Therefore, NLNs, pTNM stage and PD-L1 expression

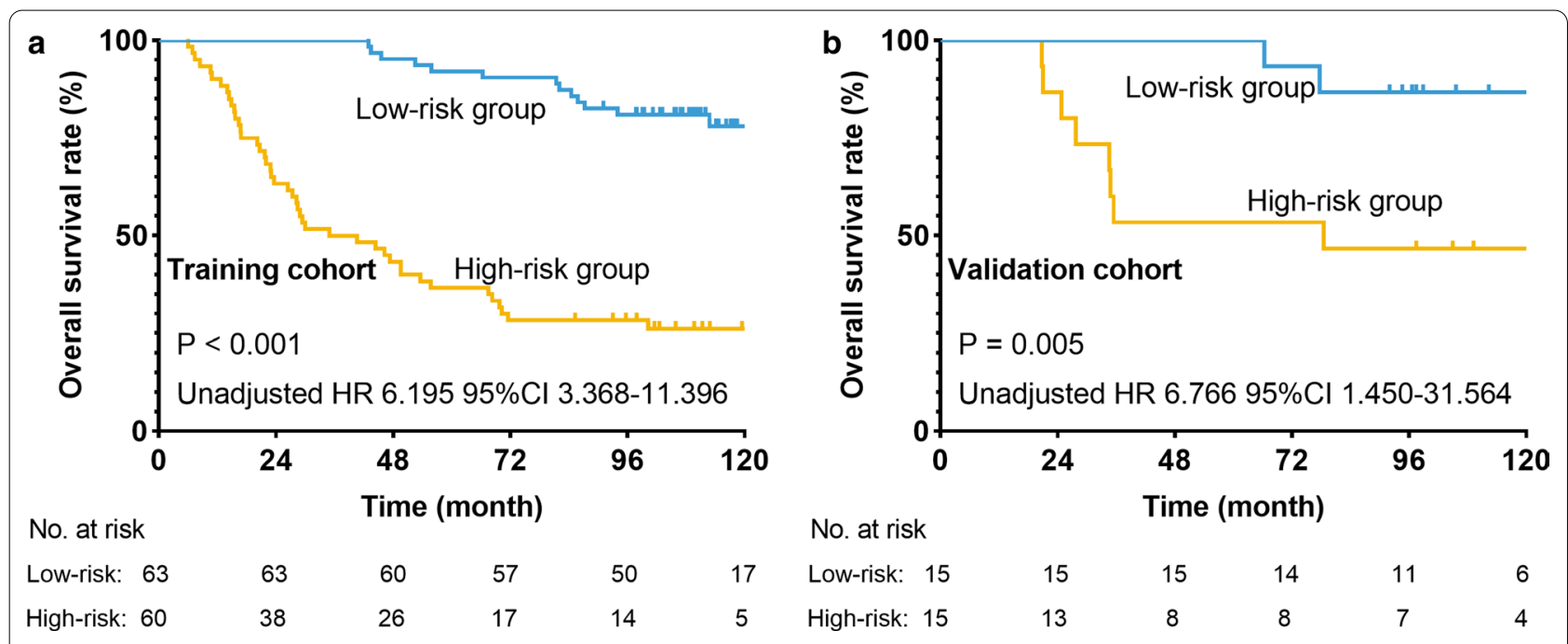

Fig. 4 Overall survival curve for cohort of patients with stage IB/IIA ESCC according to the PS (a training cohort; $\mathbf{b}$ validation cohort) 
levels can be clinically measured, thus facilitating the model's wide range of applications.

We found that the differentiation of tumor had a difference between training and validation groups after random grouping (Table 1). However, the tumor differentiation was excluded using univariable and multivariable analyses, so the tumor differentiation had no effect in building our PS (Table 3 ). This was likely to be due to the low number of cases included in the study. Accordingly, we suggested that the degree of tumor differentiation had no effect on OS because of uneven distribution and small sample size. In addition, the $\mathrm{C}$-index of validation cohort was higher than training cohort. Given the small sample size of validation group, we found that there was likely to be an impact of "overfitting" in the process of statistics. As the median of the whole data was used as the cutoff value of, which made the results of our study more objective, we used the median as the cutoff of PD-L1 expression and NLNs.

There are some limitations in the present study. First, it is a single-institution study with a small sample size. It is therefore necessary to expand the results by performing multicenter studies with larger cohorts. Since ESCC is the main pathological type in China, the present study did not include patients with adenocarcinoma. Second, given "overfitting" might affect the results of validation group and the median was regarded as cutoff value of PD-L1, NLNs and PS, more cases are needed to further explore more appropriate statistic methods and more exact results and cutoff value. Third, since only patients with stage IB/IIA ESCC were enrolled, this model cannot predict or evaluate the prognosis of patients with lymph node metastasis and can only be applied to IB/IIA ESCC patients.

\section{Conclusions}

In conclusion, the PD-L1 expression, pTNM stage and NLNs were independent prognostic indictors for ESCC in stage IB/IIA. In addition, we present a validated PS for robust clinical stratification of IB/IIA ESCC to screen subgroups with poor prognosis. The PS had a significant improvement than PTNM stage for predictive ability of 5-year OS. Our PS may provide useful information to screen out the patients of poor prognosis. However, more studies are needed to explore the effect of PS on prognosis of ESCC patients in stage IB/IIA.

\footnotetext{
Abbreviations

ESCC: Esophageal squamous cell cancer; PS: Prognostic score; NLNs: The number of lymph nodes; OS: Overall survival; SYSUCC: Sun Yat-sen University Cancer Center; HR: Hazard ratio; Cl: Confidential interval; EC: Esophageal cancer; PD-L1: Programmed death ligand-1; AJCC: American Joint Committee on Cancer; SD: Standard deviation; SE: Standard error; LNs: Lymph nodes.
}

\section{Acknowledgements}

Thank all staff from SYSUCC for their contribution in providing surveillance data and support. The authors appreciated the editing of English language in Enago (www.enago.cn). Our sincere thanks to our respected pioneer, Professor Tie-Hua Rong, who oriented us to surgical oncology. Finally, we wish to thank our patients who were willing to provide personal information for medical research and they were the best teachers for doctors.

\section{Authors' contributions}

Conception and design of the work: MGW, WLL, MQL. Provision of study materials or patients: LP, LH, ZLJ, MGW, QLH. Acquisition of data: WLL, HW, LX, QLH, MQL. Analysis of data: MGW, WLL, MQL, HW. Interpretation of data: MGW, LP, LH ZL, WLL. WLL drafted the manuscript; MGW substantially revised the manuscript. All authors read and approved the final manuscript.

\section{Funding}

This work was supported by Guangdong Esophageal Cancer Institute Science and Technology Program (No. M201916). In addition, funding body supported the purchase of antibody and editing of English language.

\section{Availability of data and materials}

Researchers interested in this study may contact the authors to obtain the clinical data of all 153 patients. We have uploaded the data of this study in the Research Data Deposit of SYUCC, and its number was RDDA2019001167 (http://www.researchdata.org.cn/).

\section{Ethics approval and consent to participate}

The study protocol was approved by the Ethics Committee of Sun Yat-sen University Cancer Center (Approval No: YB2016-070), and consent was waived.

\section{Consent for publication}

All authors agree with publication in this journal.

\section{Competing interests}

The authors declare that they have no competing interests.

\section{Author details}

${ }^{1}$ Sun Yat-Sen University Cancer Center, State Key Laboratory of Oncology in South China, Collaborative Innovation Center for Cancer Medicine, Guangzhou 510060, People's Republic of China. ${ }^{2}$ Jinling Hospital, Nanjing 210000, People's Republic of China. ${ }^{3}$ The Department of Thoracic Surgery, Sun Yat-Sen University Cancer Center, 651 Dongfengdong Road, Guangzhou 510060, People's Republic of China.

Received: 5 June 2020 Accepted: 2 February 2021

Published online: 10 February 2021

\section{References}

1. Bray F, Ferlay J, Soerjomataram I, et al. Global cancer statistics 2018:GLOBOCAN estimates of incidence and mortality worldwide for 36 cancers in 185 countries. CA Cancer J Clin. 2018;68:394-424.

2. Arnold M, Soerjomataram I, Ferlay J, Forman D. Global incidence of oesophageal cancer by histological subtype in 2012. Gut. 2015;64:381-7.

3. Arnold M, Laversanne M, Brown LM, et al. Predicting the future burden of esophageal cancer by histological subtype: international trends in incidence up to 2030. Am J Gastroenterol. 2017;112:1247-55.

4. Edgren G, Adami HO, Weiderpass E, Nyren O. A global assessment of the oesophageal adenocarcinoma epidemic. Gut. 2013;62:1406-14.

5. Abnet CC, Arnold M, Wei WQ. Epidemiology of esophageal squamous cell carcinoma. Gastroenterology. 2018;154:360-73.

6. Liang H, Fan JH, Qiao YL. Epidemiology, etiology, and prevention of esophageal squamous cell carcinoma in China. Cancer Biol Med. 2017;14:33-41.

7. Du L, Wei W. Progress research in survival analysis of esophageal squamous cell carcinoma (ESCC) in Chinese population. J Pract Oncol. 2018;32(4):1002-3070.

8. Zhou L, Zhao Y, Zheng Y, et al. The prognostic value of the number of negative lymph nodes combined with positive lymph nodes in 
esophageal cancer patients: a propensity-matched analysis. Ann Surg Oncol. 2020;27:2042-50.

9. Deng J, Weng $X$, Ye J, et al. Identification of the germline mutation profile in esophageal squamous cell carcinoma by whole exome sequencing. Front Genet. 2019;10:47.

10. Wu LL, Liu X, Huang W, et al. Preoperative squamous cell carcinoma antigen and albumin serum levels predict the survival of patients with stage T1-3NOM0 esophageal squamous cell carcinoma: a retrospective observational study. J Cardiothorac Surg. 2020;15:115.

11. Kiyozumi Y, Baba Y, Okadome K, et al. IDO1 expression is associated with immune tolerance and poor prognosis in patients with surgically resected esophageal cancer. Ann Surg. 2019;269:1101-8.

12. Huang $W, W u L$, , Liu X, et al. Preoperative serum C-reactive proteinlevels and postoperative survival in patientswith esophageal squamous cell carcinoma: a propensity score matching analysis. J Cardiothorac Surg. 2019;14:167.

13. Tseng RC, Chang JM, Chen JH, et al. Deregulation of SLIT2-mediated Cdc42 activity is associated with esophageal cancer metastasis and poor prognosis. J Thorac Oncol. 2015;10:189-98.

14. Yamamura K, Baba Y, Nakagawa S, et al. Human microbiome fusobacterium nucleatum in esophageal cancer tissue is associated with prognosis. Clin Cancer Res. 2016;22:5574-81.

15. Iwai Y, Ishida M, Tanaka Y, et al. Involvement of PD-L1 on tumor cells in the escape from host immune system and tumor immunotherapy by PD-L1 blockade. PNAS. 2002;99(19):12293-7.

16. Choueiri TK, Fay AP, Gray KP, et al. PD-L1 expression in nonclear-cell renal cell carcinoma. Ann Oncol. 2014;25:2178-84.
17. D'Angelo SP, Shoushtari AN, Agaram NP, et al. Prevalence of tumorinfiltrating lymphocytes and PD-L1 expression in the soft tissue sarcoma microenvironment. Hum Pathol. 2015:46:357-65.

18. Gao Y, Li S, Xu D, et al. Prognostic value of programmed death-1, programmed death-ligand 1, programmed death-ligand 2 expression, and CD8(+) T cell density in primary tumors and metastatic lymph nodes from patients with stage T1-4N+M0 gastric adenocarcinoma. Chin J Cancer. 2017;36:61.

19. Madore J, Vilain RE, Menzies AM, et al. PD-L1 expression in melanoma shows marked heterogeneity within and between patients: implications for anti-PD-1/PD-L1 clinical trials. Pigment Cell Melanoma Res. 2015;28:245-53.

20. Muenst S, Schaerli AR, Gao F, et al. Expression of programmed death ligand 1 (PD-L1) is associated with poor prognosis in human breast cancer. Breast Cancer Res Treat. 2014;146:15-24.

21. Topalian SL, Hodi FS, Brahmer JR, et al. Safety, activity, and immune correlates of anti-PD-1 antibody in cancer. N Engl J Med. 2012;366:2443-54

22. Zhang J, Fang W, Qin T, et al. Co-expression of PD-1 and PD-L1 predicts poor outcome in nasopharyngeal carcinoma. Med Oncol. 2015;32:86.

23. National Comprehensive Cancer Network. Esophageal and Esophagogastric Junction Cancers (Version 4.2020). https://www.nccn.org/profession als/physician_gls/pdf/esophageal.pdf Accessed 14 Aug 2020.

\section{Publisher's Note}

Springer Nature remains neutral with regard to jurisdictional claims in published maps and institutional affiliations.
Ready to submit your research? Choose BMC and benefit from:

- fast, convenient online submission

- thorough peer review by experienced researchers in your field

- rapid publication on acceptance

- support for research data, including large and complex data types

- gold Open Access which fosters wider collaboration and increased citations

- maximum visibility for your research: over $100 \mathrm{M}$ website views per year

At BMC, research is always in progress.

Learn more biomedcentral.com/submissions 